\title{
Fuzzy Adaptive Tracking Control of Uncertain Strict-Feedback Nonlinear Systems with Disturbances Based on Generalized Fuzzy Hyperbolic Model
}

\author{
Jingxuan Shi, Zhongjun Yang \\ College of Information Engineering, Shenyang University of Chemical Technology, Shenyang, China \\ Email: jingxuanshi_syuct@163.com, zhongjun_yang@163.com
}

How to cite this paper: Shi, J.X. and Yang, Z.J. (2020) Fuzzy Adaptive Tracking Control of Uncertain Strict-Feedback Nonlinear Systems with Disturbances Based on Generalized Fuzzy Hyperbolic Model. Journal of Computer and Communications, 8, 50-59. https://doi.org/10.4236/jcc.2020.810006

Received: September 16, 2020

Accepted: October 25, 2020

Published: October 28, 2020

Copyright $\odot 2020$ by author(s) and Scientific Research Publishing Inc. This work is licensed under the Creative Commons Attribution International License (CC BY 4.0).

http://creativecommons.org/licenses/by/4.0/ (c) (i) Open Access

\begin{abstract}
In this paper, a fuzzy adaptive tracking control for uncertain strict-feedback nonlinear systems with unknown bounded disturbances is proposed. The generalized fuzzy hyperbolic model (GFHM) with better approximation performance is used to approximate the unknown nonlinear function in the system. The dynamic surface control (DSC) is used to design the controller, which not only avoids the "explosion of complexity" problem in the process of repeated derivation, but also makes the control system simpler in structure and lower in computational cost because only one adaptive law is designed in the controller design process. Through the Lyapunov stability analysis, all signals in the closed loop system designed in this paper are semi-globally uniformly ultimately bounded (SGUUB). Finally, the effectiveness of the method is verified by a simulation example.
\end{abstract}

\section{Keywords}

Disturbances, Uncertain Strict-Feedback Nonlinear Systems, Adaptive

Control, Generalized Fuzzy Hyperbolic Model, Dynamic Surface Control

\section{Introduction}

As an effective tool to solve the uncertainty of nonlinear systems, fuzzy logic systems are widely used in adaptive control design [1] [2] because of their good approximation capabilities. T-S (Takagi-Sugeno) fuzzy logic controller is widely used as a nonlinear function approximator [3] because it has less learning parameters to adjust online. The adaptive T-S fuzzy adaptive control method is proposed for the pure feedback nonlinear system in [4] and the uncertain MIMO 
Block Triangular nonlinear system in [5]. Compared with T-S fuzzy model, the Generalized Fuzzy Hyperbolic Model (GFHM) proposed by [6] has many advantages. Especially for nonlinear complex systems, the calculation cost is much lower than that of T-S fuzzy model because it does not require accurate object structure. The GFHM based adaptive control for several classes of nonlinear systems is studied in [7] [8] [9].

Recently, the research on adaptive fuzzy backstepping control for nonlinear system tracking control problem has been widely reported [10] [11] [12] [13]. However, there is a problem of "computational expansion" in the traditional backstepping method. Therefore, this paper proposes a Dynamic Surface Control (DSC) method to solve this problem with the help of a first-order low-pass filter [14] [15]. In [16] [17] [18], an adaptive fuzzy DSC method based on strict feedback nonlinear systems with unmeasurable states is studied.

In addition, it is well known that the dynamic disturbance signal is an important factor that leads to the instability of the system, and even leads to the serious degradation of the control system performance. Therefore, it is very meaningful to study nonlinear systems with dynamic disturbance and uncertainty in the field of control. In [19] [20], several adaptive fuzzy control methods based on strict feedback nonlinear systems with uncertain disturbances are proposed.

Inspired by the previous studies, an improved fuzzy adaptive tracking control technique combining DSC method and GFHM approximator is proposed for a class of strict feedback nonlinear systems with dynamic disturbance signals. It not only avoids the problem of calculation expansion, but also obtains higher tracking accuracy. In addition, only one adaptive law is designed in the controller design, which greatly reduces the calculation cost. It is proved that all signals of the closed-loop system are semi globally asymptotically stable by Lyapunov method.

\section{Problem Description}

\subsection{System Description}

This paper considers the following SISO strictly feedback uncertain nonlinear systems with unknown disturbances:

$$
\left\{\begin{array}{l}
\dot{x}_{j}=g_{j}\left(\underline{\boldsymbol{x}}_{j}\right)+x_{j+1}+d_{j}(t), 1 \leq j \leq n-1, \\
\dot{x}_{n}=g_{n}\left(\underline{\boldsymbol{x}}_{n}\right)+u+d_{n}(t), \\
y=x_{1},
\end{array}\right.
$$

where $\underline{\boldsymbol{x}}_{j}=\left[x_{1}, x_{2}, \cdots, x_{j}\right]^{\mathrm{T}} \in R^{j} \quad(j=1,2, \cdots, n)$ is the state variable of the system, $u$ is the control input and $y$ is the output variable of the system. $g_{j}(\cdot)$ $(j=1,2, \cdots, n)$ is an unknown smooth nonlinear function. $d_{j}(t)$ is an unknown bounded disturbance signal satisfying $\left|d_{j}\right| \leq d_{j}^{*}$ and $d_{j}^{*}$ is a constant.

\subsection{Generalized Fuzzy Hyperbolic Model}

Because the generalized fuzzy hyperbolic model makes multiple linear transfor- 
mations on the input variables, the model has universal approximation property, that is, it can approach the actual model with arbitrary precision.

The membership functions $\lambda_{P_{x}}$ and $\lambda_{N_{x}}$ of fuzzy sets $P_{x}$ and $N_{x}$ are defined as follows:

$$
\begin{aligned}
& \lambda_{P_{x}}\left(x_{s}\right)=\mathrm{e}^{-\frac{1}{2}\left(x_{s}-\eta_{s}\right)^{2}}, \\
& \lambda_{N_{x}}\left(x_{s}\right)=\mathrm{e}^{-\frac{1}{2}\left(x_{s}+\eta_{s}\right)^{2}},
\end{aligned}
$$

where the constant $\eta_{s}>0$.

Definition 1 [6]: A given system has $n$ input variables $\overline{\boldsymbol{x}}=\left[x_{1}(t), x_{2}(t), \cdots, x_{n}(t)\right]^{\mathrm{T}}$ and output variable $y(t) . \underline{\boldsymbol{x}}=\left[\underline{x}_{1}(t), \cdots, \underline{x}_{m}(t)\right]^{\mathrm{T}}$ is defined as the generalized input variable, where $\underline{x}_{j}=x_{s}-\beta_{s i} . \quad m=\sum_{j=1}^{n} \sigma_{j}$ is the number of generalized input variables, $\sigma_{s}(s=1, \cdots, n)$ is the number of $x_{s}$ linear transformations and $\beta_{s i}\left(s=1, \cdots, n, i=1, \cdots, \sigma_{s}\right)$ is the linear transformation point of $x_{s}$. If the fuzzy rule bases used to describe the system satisfy the following conditions, they are called GFHM rule bases:

1) IF $\left(x_{1}-\beta_{11}\right)$ is $\mathcal{F}_{x_{11}}$ and $\cdots$ and $\left(x_{1}-\beta_{1 \sigma_{1}}\right)$ is $\mathcal{F}_{x_{1 \sigma_{1}}}$ and $\left(x_{2}-\beta_{21}\right)$ is $\mathcal{F}_{x_{21}}$ and $\cdots$ and $\left(x_{n}-\beta_{n 1}\right)$ is $\mathcal{F}_{x_{n 1}}$ and $\cdots$ and $\left(x_{n}-\beta_{n \sigma_{n}}\right)$ is $\mathcal{F}_{x_{n \sigma_{n}}}$ THEN

$$
y=c_{\mathcal{F}_{11}}+\cdots+c_{\mathcal{F}_{1 \sigma_{1}}}+c_{\mathcal{F}_{21}}+\cdots+c_{\mathcal{F}_{n 1}}+\cdots+c_{\mathcal{F}_{n \sigma_{n}}},
$$

where $\mathcal{F}_{x_{s i}}$ is the fuzzy subset corresponding to $x_{s}-\beta_{s i}$, including two linguistic values of $P_{x}$ and $N_{x} \cdot c_{\mathcal{F}_{s i}}$ is the output constant corresponding to $\mathcal{F}_{x_{s i}}$.

2) The output constant $c_{\mathcal{F}_{s i}}\left(s=1, \cdots, n, i=1, \cdots, \sigma_{s}\right)$ corresponds to $\mathcal{F}_{x_{s i}}$ one by one, that is, if $\mathcal{F}_{x_{s i}}$ is included in IF part, then $c_{\mathcal{F}_{s i}}$ should be included in THEN part. Instead, $c_{\mathcal{F}_{s i}}$ is not included in the THEN part. $c_{P_{j}}$ is used to replace $c_{\mathcal{F}_{s i}}^{+}$and $c_{N_{j}}$ to replace $c_{\mathcal{F}_{s i}}^{-}$.

3) There are $2^{m}$ fuzzy rules in this rule base, that is, the fuzzy input variables in the IF part include all possible positive $\left(P_{x}\right)$ and negative $\left(N_{x}\right)$ combinations, and the constant parameters in the THEN part include all the output constant combinations.

Lemma 1 [6]: If there are input variables $x$ and output variables $y(t)$ for the same system as in Definition 1, and define the generalized fuzzy hyperbolic rule base and generalized input variables according to Definition 1, and defines membership functions $P_{x}$ and $N_{x}$ as Equation (2), then the following model can be obtained:

$$
\begin{aligned}
y & =\sum_{j=1}^{m} \frac{c_{P_{j}} \mathrm{e}^{\eta_{x_{j}} \underline{x}_{j}}+c_{N_{j}} \mathrm{e}^{-\eta_{x_{j}} \underline{x}_{j}}}{\mathrm{e}^{\eta_{x_{j}} \underline{x}_{j}}+\mathrm{e}^{-\eta_{x_{j}} \underline{x}_{j}}} \\
& =\sum_{j=1}^{m} a_{j}+\sum_{j=1}^{m} b_{j} \frac{\mathrm{e}^{\eta_{x_{j}} \underline{x}_{j}}-\mathrm{e}^{-\eta_{x_{j}} \underline{x}_{j}}}{\mathrm{e}^{\eta_{x_{j}} \underline{x}_{j}}+\mathrm{e}^{-\eta_{x_{j}} x_{j}}} \\
& =\xi+\boldsymbol{B}^{\mathrm{T}} \tanh (\Phi \underline{\boldsymbol{x}})=J(\boldsymbol{x}),
\end{aligned}
$$

where $a_{j}=\frac{c_{P_{j}}+c_{N_{j}}}{2}, \quad b_{j}=\frac{c_{P_{j}}-c_{N_{j}}}{2}, \quad \xi=\sum_{j=1}^{m} a_{j}, \quad \boldsymbol{B}=\left[b_{1}, \cdots, b_{m}\right]^{\mathrm{T}}$, 
$\Phi=\operatorname{diag}\left[\eta_{x_{1}}, \cdots, \eta_{x_{m}}\right]$ where $\tanh (\Phi \underline{\boldsymbol{x}})$ is given by $\tanh (\Phi \underline{\boldsymbol{x}})=\left[\tanh \left(\eta_{x_{1}} \underline{x}_{1}\right), \cdots, \tanh \left(\eta_{x_{m}} \underline{x}_{m}\right)\right]^{\mathrm{T}}$. We call model (4) the generalized fuzzy hyperbolic model.

Lemma 2 [6]: For any continuous function $h(x)$ and any real number $\varepsilon>0$ on $U \subset R^{n}$, there exists a generalized fuzzy hyperbolic model $J(x) \in Z$ ( $Z$ is a series of fuzzy basis functions) satisfying

$$
\sup _{x \in U}|h(x)-J(x)|<\varepsilon .
$$

Remark 1 [21]: As an extension of a fuzzy hyperbolic regular basis function, we can obtain the following equivalent functions of GFHM:

$$
y=\vartheta^{\mathrm{T}} \psi(\boldsymbol{x}),
$$

where $\vartheta=\left[\xi, \boldsymbol{B}^{\mathrm{T}}\right]^{\mathrm{T}}, \quad \psi(\boldsymbol{x})=\left[1, \tanh \left(\eta_{x_{1}} \underline{x}_{1}\right), \cdots, \tanh \left(\eta_{x_{m}} \underline{x}_{m}\right)\right]^{\mathrm{T}}$. The output function $y(t)$ is linear with respect to the adjustment parameter $\vartheta$. The optimization parameters $\vartheta^{*}$ are defined as follows

$$
\vartheta^{*}=\arg \min _{\vartheta \in R^{n}}\left\{\sup _{x \in U}\left|\vartheta^{* \mathrm{~T}} \psi(\boldsymbol{x})-y\right|\right\} .
$$

\section{Design of Adaptive Fuzzy Tracking Controller}

In this section, a fuzzy adaptive DSC control method based on GFHM is designed by using the preparatory knowledge in the previous section for system (1). In addition, let the normal number $W$ be $W=\max \left\{\left\|\vartheta_{j}^{*}\right\|^{2}: j=1,2, \cdots, n\right\}$. According to backstepping, the design process of the controller includes $n$ steps.

First of all, in the DSC design, there are the following transformations:

$$
\begin{gathered}
e_{1}=x_{1}-y_{r} \\
\bar{e}_{j}=e_{j}-s_{j} \\
e_{j+1}=x_{j+1}-\bar{v}_{j+1} \\
\dot{s}_{j}=-k_{j} s_{j}+\bar{v}_{j+1}-v_{j+1}
\end{gathered}
$$

where $j=1, \cdots, n-1, \quad y_{r}$ is the output reference signal, $e_{j}$ is the tracking error, $\bar{e}_{j}$ is the tracking error of the transformation, $s_{j}$ is the design parameter, $k_{j}$ is the positive constant, $v_{j}$ is the virtual control law and $\bar{v}_{j}$ is the first-order filter signal with time constant $l_{j}>0$.

$$
l_{j} \dot{\bar{v}}_{j}+\bar{v}_{j}=v_{j}, \quad \bar{v}_{j}(0)=v_{j}(0) \text {. }
$$

where $j=2, \cdots, n$.

Then, the transformed error system is as follows

$$
\begin{aligned}
& \dot{\bar{e}}_{j}=-k_{j} \bar{e}_{j}+g_{j}\left(x_{j}\right)+d_{j}+\varepsilon_{j}-\frac{1}{2} \bar{e}_{j} \hat{W} \psi_{j}^{\mathrm{T}}\left(x_{j}\right) \psi_{j}\left(x_{j}\right)-\bar{e}_{j-1}+\bar{e}_{j+1} \\
& \dot{\bar{e}}_{n}=-k_{n} \bar{e}_{n}+g_{n}\left(x_{n}\right)+d_{n}+\varepsilon_{n}-\frac{1}{2} \overline{e_{n}} \hat{W} \psi_{n}^{\mathrm{T}}\left(x_{n}\right) \psi_{n}\left(x_{n}\right)-\bar{e}_{n-1}
\end{aligned}
$$

where $j=1, \cdots,(n-1), \bar{e}_{0}=0, \tilde{W}=W-\hat{W}$ and $\hat{W}$ is the estimated value of $W$. The equivalent function $\vartheta^{*} \psi(x)$ of GFHM is used to approximate the nonlinear function $g_{j}(\cdot)$, and the design virtual control laws $v_{j+1}$ are 


$$
\begin{gathered}
v_{2}=-k_{1} e_{1}-s_{2}+\dot{y}_{r}-\frac{1}{2} \bar{e}_{1} \hat{W} \psi_{1}^{\mathrm{T}}\left(x_{1}\right) \psi_{1}\left(x_{1}\right) \\
v_{j+1}=-k_{j} e_{j}-s_{j+1}+\dot{\bar{v}}_{j}-\frac{1}{2} \bar{e}_{j} \hat{W} \psi_{j}^{\mathrm{T}}\left(x_{j}\right) \psi_{j}\left(x_{j}\right)-\bar{e}_{j-1}
\end{gathered}
$$

where $j=2, \cdots,(n-1)$.

Finally, according to the design program, the parameter $s_{n}$ is redesigned to meet the following conditions

$$
\dot{s}_{n}=-k_{n} s_{n}
$$

Therefore, we can design controller $u$ and adaptive law according to the following formula

$$
\begin{gathered}
u=-k_{n} e_{n}+\dot{\bar{v}}_{n}-\frac{1}{2} \bar{e}_{n} \hat{W} \psi_{n}^{\mathrm{T}}\left(x_{n}\right) \psi_{n}\left(x_{n}\right)-\bar{e}_{n-1} \\
\dot{\hat{W}}=\sum_{j=1}^{n}\left(\frac{r}{2} \bar{e}_{j}^{2} \psi_{j}^{\mathrm{T}}\left(x_{j}\right) \psi_{j}\left(x_{j}\right)\right)-q \hat{W}
\end{gathered}
$$

where $r$ and $q$ are the positive constants of the design.

Remark 2: Compared with [9], this method only needs the information of $y_{r}$ and $\dot{y}_{r}$, while the common backstepping design needs the information of $y_{r}^{(i)}$ $(i=1,2, \cdots, n)$. In addition, the DSC using the first-order filter (Equation (11)) avoids the repeated derivative problem of nonlinear function $g_{j}\left(x_{j}\right)$ in the design of controller Equation (13), Equation (14) and Equation (16).

Remark 3: In this method, $\mathrm{n}$ adaptive laws need not be designed. Only one adaptive parameter needs to be adjusted, which greatly reduces the computational burden.

\section{Stability Analysis}

The stability of the designed control method is proved in this section.

Theorem 1: If there are virtual control variables such as Equation (13) and Equation (14), such as the actual control variables of Equation (16), and the adaptive law of Equation (17), then the nonlinear system (1) is semi globally uniformly ultimately bounded, and the tracking error is kept in a small range.

Proof: Define Lyapunov functional as

$$
V=\frac{1}{2} \sum_{j=1}^{n} \bar{e}_{j}^{2}+\frac{1}{2 r} \tilde{W}^{2} .
$$

The derivative of $V$ is obtained

$$
\begin{aligned}
\dot{V}= & \sum_{j=1}^{n} \bar{e}_{j} \dot{\bar{e}}_{j}-\frac{1}{r} \tilde{W} \dot{\hat{W}} \\
= & -\sum_{j=1}^{n} k_{j} \bar{e}_{j}^{2}+\sum_{j=1}^{n} \bar{e}_{j} \vartheta_{j}^{*} \psi_{j}\left(\underline{x}_{j}\right)+\sum_{j=1}^{n} \bar{e}_{j} \varepsilon_{j} \\
& +\sum_{j=1}^{n} \bar{e}_{j} d_{j}-\sum_{j=1}^{n} \frac{1}{2} \bar{e}_{j}^{2} \hat{W} \psi_{j}^{\mathrm{T}}\left(\underline{x}_{j}\right) \psi_{j}\left(\underline{x}_{j}\right)-\frac{1}{r} \tilde{W} \dot{\hat{W}}
\end{aligned}
$$

Next, we know 


$$
\begin{aligned}
\bar{e}_{j} \vartheta_{j}^{*} \psi_{j}\left(\underline{x}_{j}\right) & \leq \frac{1}{2} \bar{e}_{j}^{2} \vartheta_{j}^{* \mathrm{~T}} \vartheta_{j}^{*} \psi_{j}^{\mathrm{T}}\left(\underline{x}_{j}\right) \psi_{j}\left(\underline{x}_{j}\right)+\frac{1}{2} \\
& \leq \frac{1}{2} \bar{e}_{j}^{2} W \psi_{j}^{\mathrm{T}}\left(\underline{x}_{j}\right) \psi_{j}\left(\underline{x}_{j}\right)+\frac{1}{2} \\
\bar{e}_{j} \varepsilon_{j} & \leq \frac{1}{2} \bar{e}_{j}^{2}+\frac{1}{2} \varepsilon_{j}^{*_{2}} \\
\bar{e}_{j} d_{j} & \leq \frac{1}{2} \bar{e}_{j}^{2}+\frac{1}{2} d_{j}^{* 2}
\end{aligned}
$$

Substituting Equations (20)-(22) into Equation (19), it can be deduced that

$$
\dot{V} \leq-\sum_{j=1}^{n}\left(k_{j}-1\right) \bar{e}_{j}^{2}+\frac{1}{2} \bar{e}_{n}^{2}+\sum_{j=1}^{n} \frac{1}{2} \bar{e}_{j}^{2} \tilde{W} \psi_{j}^{\mathrm{T}}\left(\underline{x}_{j}\right) \psi_{j}\left(\underline{x}_{j}\right)+\omega_{1}-\frac{1}{r} \tilde{W} \dot{\hat{W}}
$$

where $\omega_{1}=\frac{n}{2}+\sum_{j=1}^{n} \frac{1}{2} \varepsilon_{j}^{* 2}+\sum_{j=1}^{n} \frac{1}{2} d_{j}^{* 2}$

Then substituting Equation (17) into Equation (23), we can get

$$
\begin{aligned}
\dot{V} \leq & -\sum_{j=1}^{n}\left(k_{j}-1\right) \bar{e}_{j}^{2}+\frac{1}{2} \bar{e}_{n}^{2}+\sum_{j=1}^{n} \frac{1}{2} \bar{e}_{j}^{2} \tilde{W} \psi_{j}^{\mathrm{T}}\left(\underline{x}_{j}\right) \psi_{j}\left(\underline{x}_{j}\right) \\
& +\omega_{1}-\frac{1}{r} \tilde{W}\left(\sum_{j=1}^{n}\left(\frac{r}{2} \bar{e}_{j}^{2} \psi_{j}^{\mathrm{T}}\left(\underline{x}_{j}\right) \psi_{j}\left(\underline{x}_{j}\right)\right)-k_{0} \hat{W}\right) \\
\leq & -\sum_{j=1}^{n}\left(k_{j}-1\right) \bar{e}_{j}^{2}+\frac{1}{2} \bar{e}_{n}^{2}+\omega_{1}+\frac{k_{0}}{r} \tilde{W} \hat{W}
\end{aligned}
$$

Notice the following equation

$$
\frac{k_{0}}{r} \tilde{W} \hat{W}=\frac{k_{0}}{r} \tilde{W}(W-\tilde{W}) \leq-\frac{k_{0}}{2 r} \tilde{W}^{2}+\frac{k_{0}}{2 r} W^{2}
$$

Then, by rearranging Equation (24), we can get

$$
\begin{aligned}
\dot{V} & \leq-\sum_{j=1}^{n}\left(k_{j}-1\right) \bar{e}_{j}^{2}+\frac{1}{2} \bar{e}_{n}^{2}-\frac{k_{0}}{2 r} \tilde{W}^{2}+\frac{k_{0}}{2 r} W^{2}+\omega_{1} \\
& \leq-\sum_{j=1}^{n}\left(k_{j}-\frac{3}{2}\right) \bar{e}_{j}^{2}-\frac{k_{0}}{2 r} \tilde{W}^{2}+\Lambda \\
& \leq-\Phi V+\Lambda,
\end{aligned}
$$

where $\Lambda=\frac{k_{0}}{2 r} W^{2}+\omega_{1}, \Phi=\min \left\{2 k_{j}-3, k_{0}, j=1, \cdots, n\right\}$.

From Equation (26), we can conclude that

$$
V(t) \leq\left(V\left(t_{0}\right)-\frac{\Lambda}{\Phi}\right) \mathrm{e}^{-\Phi\left(t-t_{0}\right)}+\frac{\Lambda}{\Phi} .
$$

Inequality (27) shows that $V(t)$ is ultimately bounded and the boundary value is $\frac{\Lambda}{\Phi}$. Therefore, we can consider that all the signals of the closed-loop system (i.e., $\bar{e}_{j}, j=1, \cdots, n$ and $\left.\tilde{W}\right)$ are semi global uniformly ultimately bounded.

\section{Simulation Example}

References [8] [22] considers the tracking control problem of a single joint manipulator driven by a brush DC motor, and the simulation results are verified by 
the control method designed in this paper. The nonlinear dynamic equation of the system is as follows:

$$
\left\{\begin{array}{l}
C \ddot{p}+A \dot{p}+L \sin (p)=I+d_{I} \\
Q \dot{I}+R I=-K_{m} \dot{p}+U,
\end{array}\right.
$$

where $p, \dot{p}$ and $\ddot{p}$ are the angular displacement, velocity and acceleration of the joint, respectively. $I$ is the current of the motor. $d_{I}$ is a random disturbance signal given by $d_{I}=4 \sin (t)$. $U$ is the motor input voltage. Other parameters are set as $C=1, A=1, Q=1, R=0.5, L=2.2$ and $K_{m}=5$.

Set $x_{1}=p, x_{2}=\dot{p}, x_{3}=I$, so Equation (28) can be expressed as the form of system (1), as follows

$$
\left\{\begin{array}{l}
\dot{x}_{1}=x_{2} \\
\dot{x}_{2}=-2.2 \sin \left(x_{1}\right)-x_{2}+x_{3}+4 \sin (t) \\
\dot{x}_{3}=-5 x_{2}-0.5 x_{3}+u \\
y=x_{1}
\end{array}\right.
$$

The initial state value is set as $\left[x_{1}(0), x_{2}(0), x_{3}(0)\right]^{\mathrm{T}}=[0.5,0.5,0.5]$. Set parameters $k_{1}=2.5, k_{2}=18, k_{3}=0.1, l_{2}=0.08, l_{3}=0.08, r=30, k_{0}=0.1$. $y_{r}=(\pi / 2) \sin (t)\left(1-\mathrm{e}^{-0.1 t^{2}}\right)$ is the given reference signal.

The trajectory curve of output signal $y$ tracking reference signal $y_{r}$ are shown in Figure 1. The tracking error $e$ is shown in Figure 2. The control input $u$ is shown in Figure 3. It can be seen from the simulation results that the tracking error $e$ and the control input $u$ are semi-globally uniformly ultimately bounded, and the tracking error $e$ converges rapidly to a compact set near the origin. Compared with [8], the simulation results show that the proposed method can obtain faster adaptive and higher tracking accuracy.

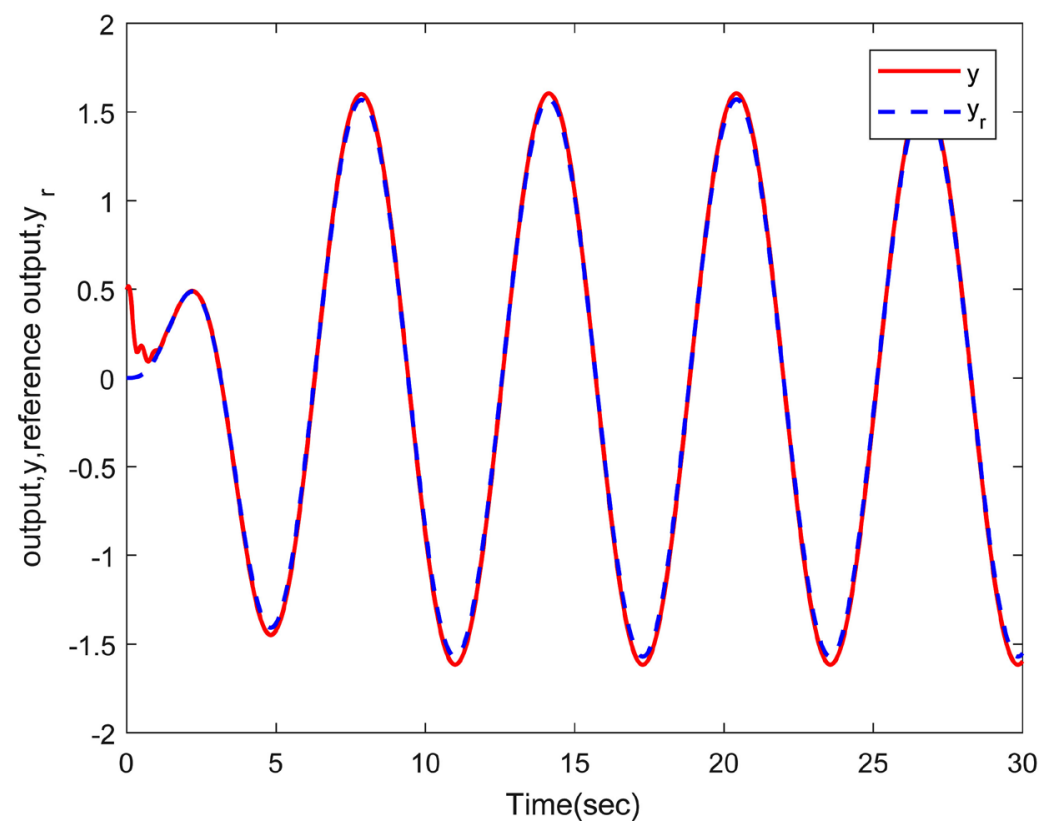

Figure 1. Output $y$ and reference output $y_{r}$. 


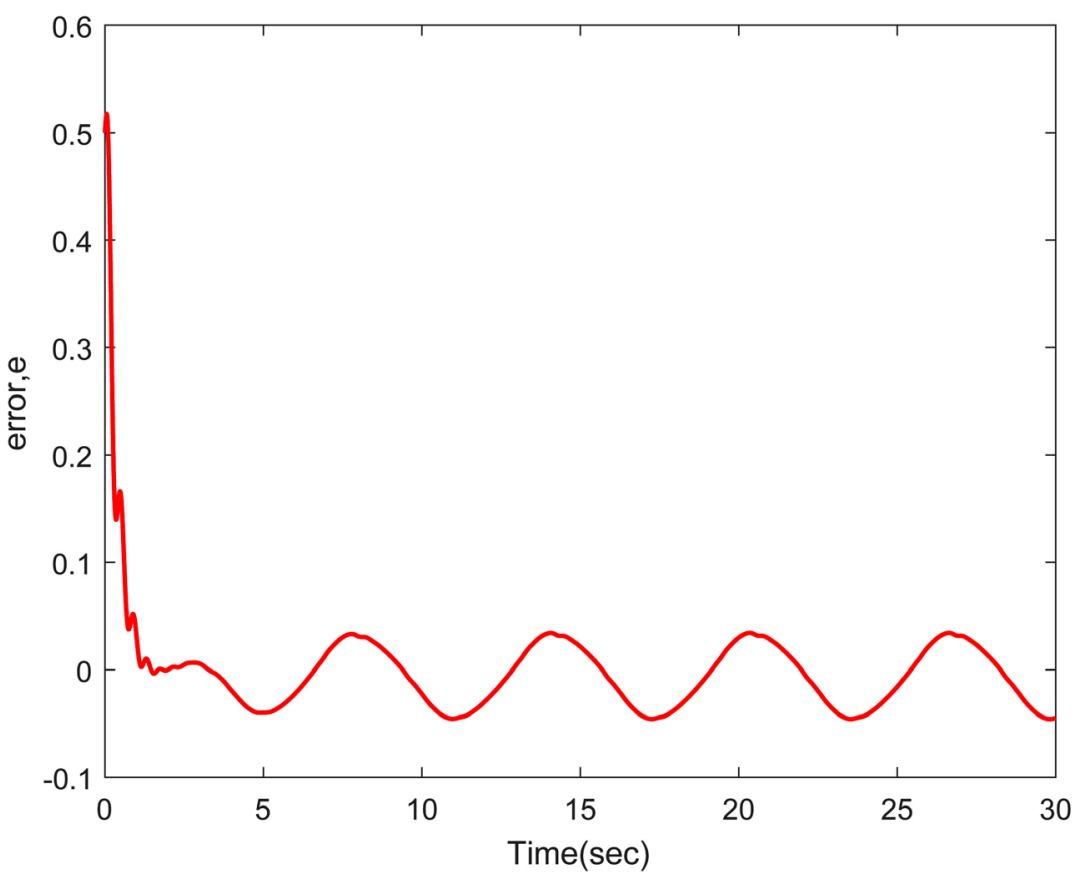

Figure 2. Tracking error $e$.

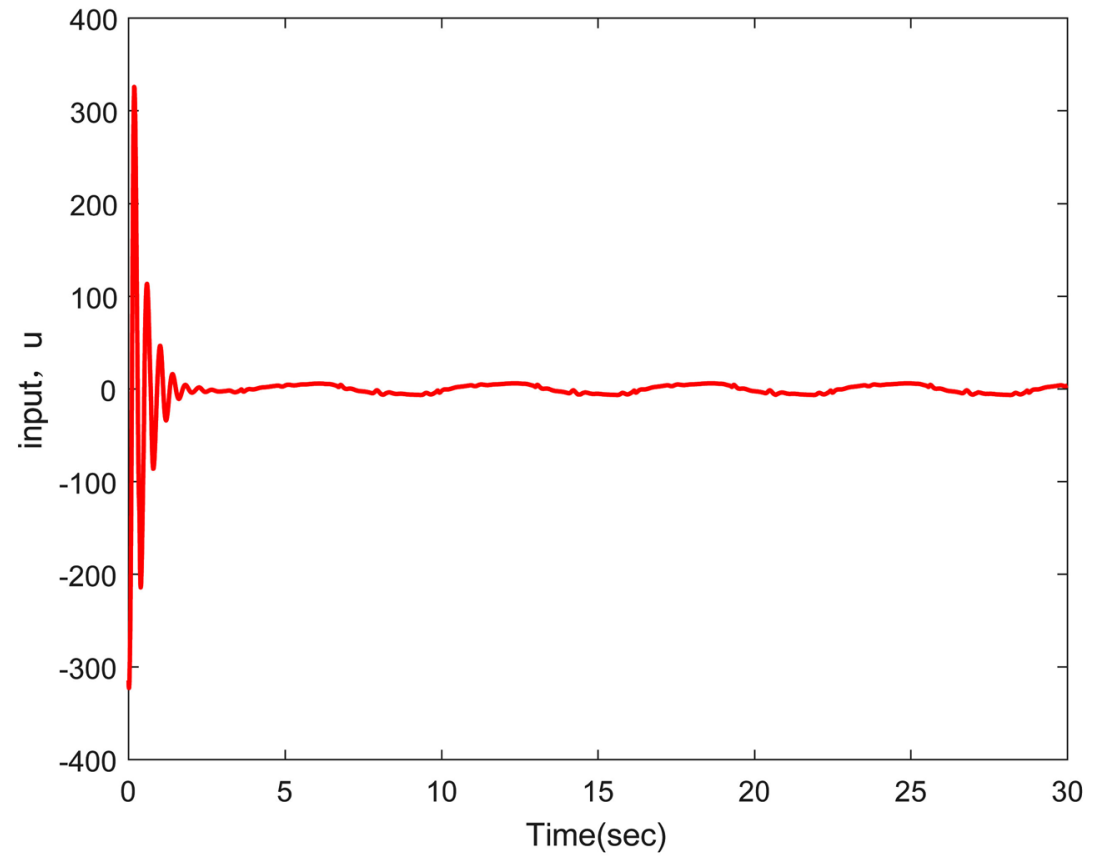

Figure 3. Control input $u$.

\section{Conclusion}

In this paper, the problem of fuzzy adaptive tracking control for a class of uncertain SISO strict feedback nonlinear systems with disturbance is studied. In this control method, GFHM nonlinear function approximator is introduced to improve the approximation performance, and DSC technology is used to obtain better tracking performance. It not only avoids the problem of calculation ex- 
pansion, but also obtains higher tracking accuracy. In addition, only one adaptive law is designed in the controller design, which greatly reduces the calculation cost. The SGUUB of the system is proved by Lyapunov stability theory. The effectiveness of the control method is further proved by simulation examples.

\section{Conflicts of Interest}

The authors declare no conflicts of interest regarding the publication of this paper.

\section{References}

[1] Wang, J.H., Liu, Z., Chen, C.L.P., Zhang, Y. and Lai, G.Y. (2019) Extended Dimension Fuzzy Adaptive Control for Nonlinear Uncertain Stochastic Systems with Actuator Constraints. Nonlinear Dynamics, 98, 1315-1329. https://doi.org/10.1007/s11071-019-05265-2

[2] Lu, Y.K. (2018) Adaptive-Fuzzy Control Compensation Design for Direct Adaptive Fuzzy Control. IEEE Transactions on Fuzzy Systems, 26, 3222-3231. https://doi.org/10.1109/TFUZZ.2018.2815552

[3] Wang, J.H., Ahn, C.K., Gao, Y.B. and Qiu, J.B. (2017) Sliding Mode Control for Non-Linear Systems by Takagi-Sugeno Fuzzy Model and Delta Operator Approaches. IET Control Theory and Applications, 11, 1205-1213. https://doi.org/10.1049/iet-cta.2016.0231

[4] Zhang, T.P., Wen, H. and Zhu, Q. (2010) Adaptive Fuzzy Control of Nonlinear Systems in Pure Feedback Form Based on Input-to-State Stability. IEEE Transactions on Fuzzy Systems, 18, 80-93. https://doi.org/10.1109/TFUZZ.2009.2036906

[5] Li, T.S., Wang, D. and Chen, N.X. (2011) Adaptive Fuzzy Control of Uncertain MIMO Non-Linear Systems in Block-Triangular Forms. Nonlinear Dynamics, 63, 105-123. https://doi.org/10.1007/s11071-010-9789-0

[6] Zhang, H.G., Wang, Z.L., Li, M., Quan, Y.B. and Zhang, M.J. (2004) Generalized Fuzzy Hyperbolic Model: A Universal Approximator. Journal of Northeastern University, 30, 416-422.

[7] Yang, Z.J., Zhang, H.G. and Cui, Y. (2018) Adaptive Fuzzy Tracking Control for Switched Uncertain Strict-Feedback Nonlinear Systems. Journal of the Franklin Institute, 355, 714-727. https://doi.org/10.1016/j.jfranklin.2017.11.038

[8] Cui, Y., Zhang, H.G., Wang, Y.C. and Gao, W.Z. (2016) Adaptive Control for a Class of Uncertain Strict-Feedback Nonlinear Systems Based on a Generalized Fuzzy Hyperbolic Model. Fuzzy Sets \& Systems, 302, 52-64. https://doi.org/10.1016/j.fss.2015.11.015

[9] Cui, Y., Zhang, H.G. and Wang, Y.C. (2017) Adaptive Tracking Control of Uncertain MIMO Nonlinear Systems Based on Generalized Fuzzy Hyperbolic Model. Fuzzy Sets \& Systems, 306, 105-117. https://doi.org/10.1016/j.fss.2016.04.011

[10] Zhou, S.S., Feng, G. and Feng, C.B. (2005) Robust Control for a Class of Uncertain Nonlinear Systems: Adaptive Fuzzy Approach Based on Backstepping. Fuzzy Sets \& Systems, 151, 1-20. https://doi.org/10.1016/j.fss.2004.05.008

[11] Wang, M., Chen, B. and Dai, S.L. (2007) Direct Adaptive Fuzzy Tracking Control for a Class of Perturbed Strict-Feedback Nonlinear Systems. Fuzzy Sets \& Systems, 158, 2655-2670. https://doi.org/10.1016/j.fss.2007.06.001

[12] Chen, Z., Huang, F.H., Yang, C. and Yao, B. (2019) Adaptive Fuzzy Backstepping 
Control for Stable Nonlinear Bilateral Teleoperation Manipulators with Enhanced Transparency Performance. IEEE Transactions on Industrial Electronics, 67, 746-756. https://doi.org/10.1109/TIE.2019.2898587

[13] Zhang, H.G., Cui, Y. and Wang, Y.C. (2017) Hybrid Fuzzy Adaptive Fault-Tolerant Control for a Class of Uncertain Nonlinear Systems with Unmeasured States. IEEE Transactions on Fuzzy Systems, 25, 1041-1050.

https://doi.org/10.1109/TFUZZ.2016.2593500

[14] Yip, P.P. and Hedrick, J.K. (1998) Adaptive Dynamic Surface Control: A Simplifified Algorithm for Adaptive Backstepping Control of Nonlinear Systems. International Journal of Control, 71, 959-979. https://doi.org/10.1080/002071798221650

[15] Swaroop, D., Hedrick, J.K., Yip, P.P. and Gerdes, J.C. (2000) Dynamic Surface Control for a Class of Nonlinear Systems. IEEE Transactions on Automatic Control, 45, 1893-1899. https://doi.org/10.1109/TAC.2000.880994

[16] Tong, S.C., Li, Y.M., Feng, G. and Li, T.S. (2011) Observer-Based Adaptive Fuzzy Backstepping Dynamic Surface Control for a Class of MIMO Nonlinear Systems. IEEE Transactions on Systems Man \& Cybernetics Part B, 41, 1124-1135. https://doi.org/10.1109/TSMCB.2011.2108283

[17] Tong, S.C., Sui, S. and Li, Y.M. (2015) Fuzzy Adaptive Output Feedback Control of MIMO Nonlinear Systems with Partial Tracking Errors Constrained. IEEE Transactions on Fuzzy Systems, 23, 729-742. https://doi.org/10.1109/TFUZZ.2014.2327987

[18] Chen, W.S., Jiao, L.C., Li, R.H. and Li, J. (2010) Adaptive Backstepping Fuzzy Control for Nonlinearly Parameterized Systems with Periodic Disturbances. IEEE Transactions on Fuzzy Systems, 18, 674-685. https://doi.org/10.1109/TFUZZ.2010.2046329

[19] Xu, B., Shi, Z.K. and Yang, C.G. (2015) Composite Fuzzy Control of a Class of Uncertain Nonlinear Systems with Disturbance Observer. Nonlinear Dynamics, 80, 341-351. https://doi.org/10.1007/s11071-014-1872-5

[20] Liu, Y.J., Tong, S.C. and Chen, C.L.P. (2013) Adaptive Fuzzy Control via Observer Design for Uncertain Nonlinear Systems with Unmodeled Dynamics. IEEE Transactions on Fuzzy Systems, 21, 275-288. https://doi.org/10.1109/TFUZZ.2012.2212200

[21] Zhang, M.J., Shao, C. and Li, S.K. (2012) Robust Indirect Adaptive Fuzzy Control Based on the GFHM. International Conference on System Science \& Engineering, 248-252.

[22] Li, T.S., Wang, D., Feng, G. and Tong, S.C. (2010) A DSC Approach to Robust Adaptive NN Tracking Control for Strict-Feedback Nonlinear Systems. IEEE Transactions on Systems Man \& Cybernetics Part B Cybernetics A Publication of the IEEE Systems Man \& Cybernetics Society, 40, 915-927. https://doi.org/10.1109/TSMCB.2009.2033563 